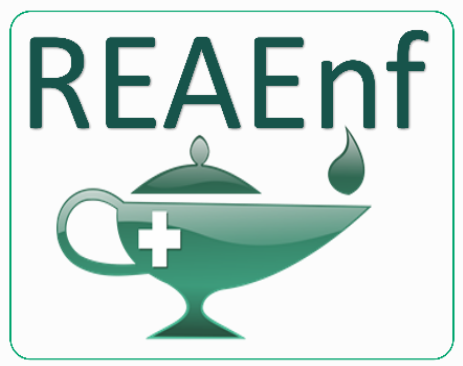

Revista Eletrônica Acervo Enfermagem
REVISÃO BIBLIOGRÁFICA

Recebido em: 9/2020

Aceito em: 10/2020

Publicado em: 11/2020

\title{
Abordagem da enfermagem nos cuidados paliativos oncológicos em tempos de pandemia do covid-19: revisão narrativa para contribuição científica
}

Nursing approach in palliative oncological care in pandemic times of covid-19: narrative review for scientific contribution

Enfoque de enfermería en la atención oncológica paliativa en tiempos de pandemia de covid-19: revisión narrativa para la contribución científica

Fábio Manoel Gomes da Silva ${ }^{1 *}$, Vanessa Kelly Cardoso Estumano ${ }^{2}$, Vanessa Pompeu Baia Miranda ${ }^{3}$, Tainá Priscila dos Santos Bezerra ${ }^{4}$, Raimunda Ferreira de Sousa ${ }^{4}$, Zenon Rodrigues de Sousa ${ }^{4}$, Rosilene da Silva ${ }^{5}$.

Resumo: Realizar uma revisão bibliográfica analisando o poder de decisão do paciente oncológico que, em leito de terapia intensiva, em um cenário de pandemia do novo coronavirus com a importância da preservação das faculdades mentais plenas ou parciais nos últimos dias de vida, manifesta se interesse por descontinuar o tratamento em leito de UTI pela possibilidade eminente de mais um agravante do contagio do covid-19 (coronavirus). O presente conteúdo científico e narrativo reverencia a atuação da assistência multiprofissional dentro do atual contexto da humanização de um paciente dependente de outros, por considerar os últimos desejos quanto conscientes, não restringindo se somente aos procedimentos clínicos oncológicos. Espera se que através deste conteúdo, os profissionais de enfermagem possam ter maior aprimoramento na atuação holística do paciente oncológico, bem como na capacitação de orientar seus familiares e amigos a desenvolver abordagem confortantes ao mesmo em período de isolamento social. Observou-se que através desta investigação pode se traçar um cenário muito importante no que tange a abordagem a esta referida clientela em fase terminal, ressaltando as recentes mudanças na assistência em Unidades de Terapia Intensiva, oncologia e novas perspectivas da terapêutica do novo covid-19.

Palavras-chave: Enfermagem, Cuidados paliativos, Cuidados críticos.

Abstract: Execute a bibliographic revision analyzing the power of decision of oncologic patient that, on intensive bed therapy, in a scenario of pandemy of the new coronavirus with the importance of preservation of full mental faculties or partials in the last days of life, display to himself desire for discontinue the treatment on

\footnotetext{
1 Universidad Europea del Atlântico. Belém - PA.

2 Universidade Federal do Pará (UFPA). Belém - PA.

3 Escola Superior da Amazônia (ESAMAZ). Belém - PA.

${ }^{4}$ Universidade da Amazônia (UNAMA). Belém - PA.

${ }^{5}$ Universidade Brasil. Belém - PA.
} 
bed of UTI for the eminent possibility of a more aggravating of contagion from covid-19 (coronavirus). The present scientific content and narrative praise the acting of assistance multi-professional inside of current context of humanization of a patient dependent to others, for regard the last wishes as conscious ones, do not restricting if only to the oncologic clinic procedures. It is expected that through this content, the nursing professionals can have great enhancement in the holistic acting of the oncologic patient, as well as in the capacitation to guide their relatives and friends to develop comforting approach even in period of social isolation. It was observed that by this investigation might be draw a scenario very important in reference of the approach to this referred customers in the terminal phase, highlighting the recent changes in assistance in intensive therapy Units, oncology and new perspectives of the therapeutics of the new covid- 19 .

Keywords: Nursing, Paliative cares, Critic cares.

Resumen: Realizar una revisión bibliográfica analizando el poder de decisión del paciente oncológico que, en lecho de terapia intensiva, en un escenario de pandemia del nuevo coronavirus con la importancia de la preservación de las facultades mentales plenas o parciales en los últimos días de vida, se manifiesta el interés por descontinuar el tratamiento en lecho de UTI por la posibilidad eminente de más un agravante del contagio del covid-19 (coronavirus). El presente contenido científico y narrativo reverencia la actuación de la asistencia multiprofesional adentro del actual contexto de humanización de un paciente dependente de otros, por considerar los últimos deseos cuanto conscientes, sin restricción se solamente los procedimientos clínicos oncológicos. Se espera que, a través de este contenido, los profesionales de enfermería puedan tener mayores aprimoramiento en la actuación holística del paciente oncológico, bien como la capacitación de orientar sus familiares y amigos a desarrollar abordaje confortable al mismo en tiempo de aislamiento social. Se há observado que a través de esta investigación puede caminar a un escenario muy importante que alcanza la abordaje a esta referida clientela en fase terminal, resaltando los recientes cambios en la asistencia en Unidades de Terapia Intensiva, oncología e nuevas perspectivas de la terapéuticas del nuevo covid-19.

Palabras clave: Enfermeria, Cuidados paliativos, Cuidados críticos.

\section{INTRODUÇÃO}

Frente aos aspectos sociais os quais o ser humano se depara com situações em que as decisões se tornam importantes e essenciais na vida de outros, seja ente querido ou amigos. Uma delas é a inserção contextual da palavra câncer associado com a realidade do novo coronavírus, no convívio diário dos cidadãos que na sua grande totalidade, encontrava se em harmonia perante suas atividades diárias e habituais. $\mathrm{O}$ câncer é uma palavra não muito aceita por demonstrar aspectos do fim de vida, visto que alguns veículos de comunicação deturpam o que tange diagnóstico e tratamento, no qual a busca incessante de um tratamento fidedigno ou uma cura, demonstra anseios na questão do real foco delicado a ser acolhido no momento do diagnóstico e do ambiente hospitalar encontrando se inseridos estes pacientes (CEZAR VS, et al., 2019).

A patologia citada, pode de certa forma cogitar em cura total ou remição dependendo de tratamentos inovadores da medicina atual, ou na corrida cientifica na busca da cura através de pesquisas com células troncos, eventualmente com o tropismo por algum sistema ou tecido em específico, dependendo sempre de procedimentos invasivos como biópsias e punções para elucidar o tipo de célula acometida, e traçar conforme a equipe especializada, sendo médicos ou enfermeiros, o melhor e o real tratamento voltado para proporcionar uma grande probabilidade de reestabelecimento físico e mental para alguns pacientes, nunca deixando de repassar informações essenciais para evitar expectativas acima do esperado no momento conturbado de uma pandemia (OLIVEIRA DSA, et al., 2019).

Os anseios da expansão do novo coronavirus pode influenciar de forma significativa na decisão de persistir em querer deixar o ente querido internado em um leito de terapia intensiva. Sabendo se que diante do comprometimento do sistema imunológico, este pode em algum momento durante a permanência em um ambiente hospitalar ter contato direto com o covid-19, tornando se talvez fatal, antecipando a morte sem a real necessidade, por complicações inerentes a ação do contágio viral. Considerando se também a 
interrupção avassaladora do luto propriamente dito no pós óbito imediato, por se tratar do possível contagio concomitante deste vírus devido as exigências de protocolos que precisam ser executados por medidas preventivas para não haver contaminação entre familiares, mantendo o caixão lacrado sem o menor contato e aproximação de familiares, trazendo traumas psicológicos talvez irreversíveis (PAIVA DR, 2018).

Desde que o novo coronavirus surgiu no mundo a atenção está toda voltada para o acolhimento, terapêutica e possível cura diante de estudos precisos por toda comunidade cientifica mundial, dedicada a investigação da ação deste vírus nunca antes vivenciado no cotidiano da ação de profissionais da área da saúde, principalmente enfermeiros, que encontram se na frente da batalha biológica, enfrentamento este com poucas armas que há no mercado hospitalar, sejam fármacos ou Equipamentos de Proteção Individual (EPl's), tornando se importantes recursos no combate ao covid-19, não deixando de ser relevante o atendimento e tratamento ao paciente oncológico, mas na atual realidade recursos precisaram de fato ser destinados quase por exclusividade para o tratamento a pacientes críticos do novo coronavirus (SILVA MM, et al., 2015).

O câncer na maioria dos casos clínicos desestabiliza de maneira emocional um paciente acometido, imagina se tal diagnostico em um cenário obscuro onde todo o planeta está voltado para a procura de um tratamento ou cura de outra grave e não menos relevante doença, o novo coronavirus, principalmente ao desconhecer a maneira ideal com pequenos impactos pessoais na tomada de difíceis decisões a respeito de melhor proceder diante dos últimos dias do paciente em fase terminal, contrapondo a permanência deste em ambiente hospitalar na possibilidade deste vírus no qual estudiosos encontram se empenhados em descobrir uma cura ou tratamento. Assim, é o papel do enfermeiro, facilitar e estimular gradativamente a aceitação da condição do paciente fomentando o acolhimento e conforto de familiares, evitando ou minimizando a frustração e insatisfação da perspectiva perda deste indivíduo (CEZAR VS, et al., 2019).

A relevância para a comunidade cientifica acerca de uma temática pertinente e essencial, se observa então, o interesse profissional no comprometimento com o melhor para este paciente. O domínio da oncologia em geral consolida espaço ao enfermeiro, no quesito aperfeiçoamento de decisões e atualizações de procedimentos e técnicas em prol de traçar uma boa sistematização da assistência de enfermagem. O dilema do paciente em cuidados paliativos na Unidade de Terapia Intensiva (UTI) é conflituoso, principalmente em um cenário nebuloso da real necessidade de ocupação de leitos pelo novo coronavirus. Não obstante, também para equipe multiprofissional, em manter o processo paliativo é de total necessita da decisão conjunta perante a equipe Intensivista e familiares, em conformidade de suas reais perspectivas para o futuro. E mediante a fase paliativa é esperado aguardar o processo de degradação e falência múltipla de órgãos progressivamente proveniente da evolução metastática (SILVA MM, et al., 2015).

As Organizações Não Governamentais (ONG's) exercem um papel fundamental no acolhimento multiprofissional de familiares no conflito e no direcionamento na decisão de permanecer com o seu ente querido em um leito de terapia intensiva em estágio de cuidados proporcionais. Tais entidades sem fins lucrativos dispõe de equipe treinada e capacitada em oferecer serviços de escuta psicossocial, enaltecendo a real importância de estar com este paciente em um momento de isolamento social diante da pandemia vivenciada em todos os continentes do planeta, viabilizando desta forma momentos agradáveis para este paciente oncológico no convívio dos demais familiares nos últimos momentos (PAIVA DR, 2018).

Familiares que vivenciam o processo de doença que abrevia a morte, o câncer, combate com a desestruturação, que requer organização emocional, pois trivialmente, através das limitações dos pacientes oncológicos internados na UTI, estes estão susceptíveis e limitados, e as tomadas de decisões de permanência nesse período de terminalidade no leito, a considerar também o julgamento da equipe psicossocial que esteja acompanhando o caso clinico, traçando um parâmetro da situação familiar na possibilidade de permanecer no domicilio, necessitando de uma avaliação holística, conduta ética e decisão definitiva terapêutica. Destacando se 0 as características clínicas e fisiopatológicas peculiares e real exposição ao covid-19, podendo necessitar de Suporte Avançado de Vida (SAV). A função da terapia intensiva é de suporte terapêutico ao paciente nos períodos de maior risco ou gravidade, e não essencialmente como medidas terapêuticas paliativas (VALE TD e GARCIA PC, 2018). 
Familiares detém o poder de decisão quando o paciente que não dispõe dessas habilidades conforme suas faculdades mentais, ainda tratando se de pacientes oncológicos debilitados que apresentam quadro de demência. No entanto, considerando se os pacientes oncológicos que possuem a capacidade da tomada de decisão quanto a sua permanência ou não em um leito de UTI em seus últimos dias de vida, a decisão da equipe de saúde, que vem traçando um parâmetro deste indivíduo, em relação ao seu quadro clínico, necessitando de uma avaliação integrada, respeitando sempre aspectos legais e humanos na assertiva de priorizar as preferências do paciente e medidas de conforto, tentando ao máximo não afasta lo de seus familiares seguindo protocolos adotados pela Organização Mundial de Saúde acerca do isolamento social (SILVA MM, et al., 2015).

Diante do contexto, observar-se que uma específica classe de profissionais se torna envolvidos e comprometidos em dedicar-se a fazer o melhor na assistência desses pacientes, equipe essa que encontram se em ascensão, ou seja, profissionais paliativistas. Dessa forma, a oncologia com o decorrer dos anos está tomando um espaço íntimo para os profissionais de enfermagem, no quesito aperfeiçoamento de condutas e atualizações de procedimentos e técnicas em prol do paciente com esta referida patologia (RIBEIRO WA, et al., 2019).

Constata-se que quando ocorre o diagnóstico de metástase, o cuidado torna-se exclusivamente paliativo, devendo este paciente na atual realidade da pandemia do novo covid-19, uma vez que o paciente se encontra fora da possibilidade terapêutica de cura, devendo o profissional de saúde promover o controle de sinais e sintomas advindos do processo de degradação da doença. Desta forma, é importante preservar a dignidade, bem como os seus valores e crenças, já que a terapia para a cura não é mais eficaz.

\section{REVISÃO BIBLIOGRÁFICA}

Através do exposto conteúdo bibliográfico, profissionais podem ter um olhar amplo quanto abordar amigos e familiares no percurso de trazer conforto nos últimos dias de um paciente oncológico, pois sabe-se que através de estudos já discutidos em outras publicações em que a melhor forma de acompanhar o processo de morte é estar presente junto a pessoas queridas, isso traz um paradigma quanto a segurança do possível contagio do novo coronavirus. Certas condutas de algumas equipes multiprofissionais, no qual apenas leva se em consideração o estado clínico deste paciente, não levando em consideração seu poder de decisão e o estado emocional (MENEZES MS e FIGUEIREDO MGMCA, 2019).

A temática em questão agrega se um conteúdo bibliográfico fazendo se necessário, pois trata se de duas áreas que andam paralelamente em prol do bem-estar do paciente crítico. Estatísticas evidenciam que 0 índice de câncer nas grandes regiões metropolitanas aumentou significativamente com taxas alarmantes, aproximadamente em torno de $100 \%$ do aparecimento de novos casos, índices esses contabilizados no ano de 2018 , dentre esses $70 \%$ de novos casos notificados ocorrendo nas grandes capitais, principalmente em nível de alta complexidade, imagina se tais estatísticas agregadas aos números expressivos do covid-19, no qual pode influenciar diretamente no quantitativo de óbitos notificados no país (FABIANI L, et al., 2019).

Observa-se que na sociedade contemporânea há uma irreal imagem do que venha ser a ideia de cuidados paliativos. Constata-se que os pacientes em fase final de vida são muitas vezes abandonados em leitos de UTI, sem receber visita de seus familiares, enquanto deveriam ser acolhidos e confortados por seus entes queridos neste momento de fragilidade da vida humana, acompanhando os sinais de dor e necessidades fisiológicas diárias, pois dependendo da realidade deste paciente, pode se adquirir outros transtornos de aspecto psíquicos, como agravos de cunho psiquiátrico, ressaltando ideias de suicido e eutanásia (SILVA MM, et al., 2015).

O enfermeiro é diariamente questionado a refletir sobre o real significado da vida e da morte, e até que ponto se pode avançar nos procedimentos de suporte vital. Uma parcela de pacientes que são internados em UTI talvez necessite de fato de uma avaliação e abordagem paliativista de fim de vida desde o momento da sua admissão, a fim de que se possa estabelecer limites terapêuticos e evitar falsas esperanças aos familiares. E a equipe que assiste esse paciente, evitando um colapso do atendimento hospitalar em 
decorrência da necessidade efetiva da ocupação de leitos por pacientes acometidos pelo novo coronavirus (GULINI JEHMB, et al., 2018).

A família é entendida como uma instituição social que, quando um de seus integrantes adoece, os outros membros se reorganizam no ciclo familiar, oferecendo um suporte de apoio nesse processo vivenciado pelo paciente. A necessidade de se aprofundar acerca dos conhecimentos sobre cuidados paliativos em fase final de vida tem se tornado cada vez mais relevante no ambiente de terapia intensiva, logo, a literatura recente sugere diferentes concepções do momento da morte propriamente dita e os limites das intervenções que os profissionais de saúde Intensivista devem reconhecer e respeitar, no entanto, é necessário reconhecer no seu interim esta questão, identificando as concepções sobre o tema, e refletindo sobre a ótica do cuidado diante do processo paliativista (SILVA MM, et al., 2015).

Este fato é percebido pelos acompanhantes desses pacientes em questão, que se revezam para se manterem ao lado do seu ente querido. Além disso, diante do prognóstico de morte, cria-se a prioridade de dar suporte às necessidades físicas, emocionais e espirituais dos familiares. Dessa forma, é muito importante a participação da família no cuidado dos pacientes em fase da cessação da vida, e é a função da equipe de saúde, introduzi-la no planejamento dos cuidados primordiais, visto que a inclusão desses familiares no planejamento da assistência é um dos pressupostos do cuidado integral (PRATES DR, et al., 2019).

Destaca-se ainda que há muito o que fazer no sentido de orientar a sociedade em compreender que o morrer com dignidade é uma decorrência do viver dignamente e não meramente sobrevivência, neste entendimento, o desafio ético é considerar a questão da dignidade do fim da vida, para além do contexto médico-hospitalar e para além da dimensão fisiopatológica (FREITAS GCC e CARREIRO MA, 2018).

O seguimento de protocolos instituídos pelos órgãos competentes de saúde, precisam de certa forma serem seguidos, pois a frente da expansão do novo coronavirus, faz se de total relevância que haja um consentimento da família ou de responsáveis legais de permanecer em isolamento conjunto com seus integrantes, diante do prognostico de um paciente em cuidados paliativos. Visto que além de ocupar um possível leito de UTI, as vezes pela persistência de alguns familiares, talvez pela total falta de informação ou pelo simples fato da burocracia de poder sepultar seu ente querido, em situações que este pode evoluir a óbito na própria residência, seguindo o longo caminho de registrar um Boletim de Ocorrência (BO) em uma Delegacia e posteriormente acionar o Instituto Médico Legal (IML) para a remoção do corpo e posteriormente para o sepultamento, causando transtorno para todos os envolvidos (GULINI JEHMB, et al., 2018).

Existe a necessidade de traçar um cenário de abordagem a pacientes em fase terminal, uma vez que, são necessárias mudanças no contexto da assistência em UTI, da oncologia e dos cuidados paliativos, no qual advém da avaliação global de profissionais especialistas, pois estas áreas são importantes no prognóstico, além de ser primordial para decisões na conduta de enfermeiros quanto na total relevância do desejo ou não de um paciente oncológico em fase terminal, permanecendo ou não em um leito de UTI dentro do cotidiano de pandemia (GULINI JEHMB, et al., 2018).

A etapa de adaptação dos cuidados paliativos em algumas ocasiões são recebidas de forma positiva perante familiares, e até mesmo do próprio paciente, pois parte da prerrogativa de perspectivas não muito boas diante de uma realidade vivenciada pela pandemia do novo coronavirus, sabendo se que profissionais encontram se diante de uma batalha a um inimigo invisível e letal, o novo coronavirus, tratando se de um patógeno que ainda não há tratamento preconizado pelas entidades medicas, inviabilizando a internação deste referido paciente em leitos que possivelmente possa ser ocupados por um paciente acometido pelo covid-19, principalmente com bom prognostico de recuperação (FREITAS GCC e CARREIRO MA, 2018).

\section{CONSIDERAÇÕES FINAIS}

A análise e síntese de duas áreas, faz se necessárias pelo aspecto da sensibilização de muitos familiares desprovidos de total informação quanto a cuidados paliativos, em consideração a temática inserida acerca da pandemia do novo coronavirus, visto que um paciente encontra-se em diagnóstico de cuidados paliativos ou proporcionais de fim de vida, independente da doença de base, estes deveriam sim ter o entendimento que 0

REAEnf/EJNC | Vol. 5 | e5162 | DOI: https://doi.org/10.25248/REAenf.e5162.2020 Página 5 de 6 
fim de vida deve ser ao lado de todos aqueles que proporcionaram e objetivaram momentos agradáveis, e não apenas levar em consideração aspectos relacionados a doenças, como os agravos, trazendo para a sociedade a reflexão de humanidade e visão holística, e respeitando o desejo essencial do paciente acometido de câncer em permanecer ao lado dos familiares, principalmente por prevenção deste paciente no contagio do covid-19 no ambiente hospitalar.

\section{REFERÊNCIAS}

1. CEZAR VS, et al. Permanente em Cuidados Paliativos: uma Proposta de Pesquisa-Ação. J. res.: fundam. care. online 2019; 11 (n. esp): 324-332.

2. FABIANI L, et al. Influência da presença de metástase no perfil de mortalidade de pacientes oncológicos.REAS/EJCH | Vol.11(5)|e345.

3. FREITAS GCC, CARREIRO MA. Cuidados paliativos na Unidade de Terapia Intensiva: a ética na assistência do enfermeiro intensivista. Revista Pró-UniverSUS. 2018 Jan./Jun.; 09 (1): 86-92.

4. GULINI JEHMB, et al. Predictors of death in an Intensive Care Unit: contribution to the palliative approach. Rev Esc Enferm USP. 2018; 52: e03342.

5. MENEZES MS, FIGUEIREDO MGMCA. O papel da sedação paliativa no fim da vida: aspectos médicos e éticos Revisão. Rev. Bras. Anestesiol. vol.69 no.1 Campinas Jan./Feb. 2019.

6. OLIVEIRA DAS, et al. Sentimentos de Pacientes em Cuidados Paliativos sobre Modificações Corporais Ocasionadas pelo Câncer. Psicologia: Ciência e Profissão 2019, 39, e176879, 1-13.

7. PAIVA DR, et al. Correlação entre desempenho funcional e o tempo de permanência de pacientes neurocirúrgicos na unidade de terapia intensiva. Rev Pesq Fisio, Salvador, 2018; 8(2):167-174.

8. PRATES DR, et al. Cuidados Paliativos: Percepção de enfermeiros que atuam em Unidade de Terapia Intensiva. Rev Atenas Higeia 2019; 1(2).

9. RIBEIRO WA, et al. O enfermeiro e a implementação do cuidado ao familiar do cliente com câncer. Revista PróuniverSUS. 2019; 10(1): 86-91.

10. SANGALI AC, et al. Compreensão da família do paciente oncológico sobre cuidados paliativos, Arquivo de Ciências da Saúde, 2014; 21(3): 76-80.

11. SILVA MM, et al. Cuidados paliativos na assistência de alta complexidade em oncologia: percepção de enfermeiros. Escola Anna Nery Revista de Enfermagem. 2015; 19(3).

12. VALE, TD, GARCIA PC. Critérios de admissão do paciente oncológico em Unidades de Terapia Intensiva de hospitais gerais. Revista Ciências Médicas, 2018; 27(2): 73-84. 